\title{
Blind Cyclostationary Carrier Frequency and Symbol Rate Estimation for Underwater Acoustic Communication
}

\author{
Zhiqiang $\mathrm{Wu}^{1}$ and T. C. Yang ${ }^{2}$ \\ Department of Electrical Engineering, Wright State University ${ }^{1}$ \\ Inst. of Applied Marine Physics and Undersea Tech., Nat. Sun Yet-Sen Univ. ${ }^{2}$
}

\begin{abstract}
In this paper, we propose a new method for blind carrier frequency and symbol rate estimation based on second order cyclostationary features for underwater acoustic communication. Followed by a coarse carrier frequency and symbol rate estimation via spectral analysis, a cyclostationary based estimation algorithm is developed to provide high resolution estimation of both parameters. Specifically, a short time varying resolution spectrum correlation function and spectrum coherence function algorithm is developed to obtain spectral lines in cyclic frequency domain to accurately estimate (1) varying carrier frequency and Doppler shift caused by relative motion and (2) symbol rate for single carrier communication. Simulations confirm the effectiveness, fast speed and low complexity of the proposed algorithm. The proposed method does not assume any a priori knowledge of the target signal.
\end{abstract}

\section{INTRODUCTION}

Cyclostationary analysis has been accepted as an important tool to perform signal detection, signal parameter estimation, and modulation detection of radio frequency (RF) signals [1][8]. Cyclostationary analysis is based on the fact that communications signals are not accurately described as stationary, but rather more appropriately modeled as cyclostationary. While stationary signals have statistics that remain constant in time, the statistics of cyclostationary signals vary periodically. These periodicities occur for signals of interest in well defined manners due to underlying periodicities such as sampling, modulating, multiplexing, and coding. This resulting periodic nature of signals can be exploited to detect the existence of the signal, estimate important parameters of the signal, and determine the modulation scheme of the unknown signal.

However, due to the extremely complex and dynamic environment of underwater acoustic communication, it is not clear if the cyclostationary analysis is still applicable in these tasks. The underwater acoustic channels are extremely complex and dynamic [9]-[12]. The underwater acoustic communication signals experience severe Doppler shift, multi-path effect, phase noise and variation over time. As a direct result, the cyclostationary analysis faces significant challenges in analyzing underwater acoustic communication signals.

In this paper, we apply cyclostationary analysis to simulated data obtained by convolving the measured time-varying channel impulse with the transmitted symbols; we also include the symbol phase variations as found in the data [12]. We then estimate the signal parameters such as the carrier frequency including the Doppler shift, and symbol rate. As shown previously, the Dopper shift for a moving source can vary rapidly with time [12]. As a result, the conventional analysis using the second order spectral correlation function (SCF), which treats the channel as time invariant, does not always work. To estimate the fast varying Doppler shift, one must apply the cyclostationary analysis to short segments of the signal. To observe the spectral lines in cyclic frequency, very high cyclic frequency resolution is required which demands a high sampling rate. To overcome the computational burden in obtaining high frequency resolution, a dynamic varying resolution algorithm is adopted where high resolution is obtained only around the cyclic frequencies of interest. We note that most of the signal parameter estimation methods reported in the literature do not consider extensive multipaths (frequency selective fading) as found in the underwater channel. While the multipaths are expected to modify the features of the SCF, we find, based on our analysis, that the effect of multipaths can be minimized using the spectral coherence function (SOF) as suggested before [1], [2], [8]. As a result, the proposed method using the high resolution SOF is shown to be able to blindly estimate the carrier frequency (and Doppler shift) and symbol rate of the signal.

The rest of the paper is organized as follows: In section II, we present the system model, the cyclostationary analysis. Section III describes the short time dynamic varying resolution SCF and SOF algorithm. Section IV shows numerical results of the estimation of carrier frequency and symbol rate via simulated data.

\section{System Model AND CyClOStATIONARY ANALYSIS}

\section{A. Cyclostationary Analysis and SCF/SOF}

It is well known that most of communication signals are cyclostationary, and cyclostationary features such as spectral correlation function (SCF) and spectral coherence function (SOF) can be used in signal detection, parameter estimation and modulation detection. A signal $x(t)$ is considered to be cyclostationary in wide sense if

$$
m_{x}\left(t+T_{0}\right)=m_{x}(t)
$$




$$
R_{x}\left(t+T_{0}, u+T_{0}\right)=R_{x}(t, u)
$$

where $T_{0}$ is the period of mean $m_{x}$ and autocorrelation $R_{x}$ of $x(t)$. We can rewrite (2) in Fourier series as [1] [3]:

$$
\begin{aligned}
R_{x}(t, \tau) & =R_{x}(t+\tau / 2, t-\tau / 2) \\
& =E\left\{x(t+\tau / 2) x^{*}(t-\tau / 2)\right\} \\
& =\sum_{\alpha} R_{x}^{\alpha}(\tau) e^{j 2 \pi \alpha t}
\end{aligned}
$$

where $\alpha$ is the cyclic frequency from which features can be extracted for identifying transmitted signals [4], and $R_{x}^{\alpha}(\tau)$ denotes the cyclic autocorrelation function (CAF):

$$
R_{x}^{\alpha}(\tau)=\lim _{T \rightarrow \infty} \frac{1}{T} \int_{-T / 2}^{T / 2} R_{x}(t, \tau) e^{-j 2 \pi \alpha t} d t .
$$

The Fourier transform of the CAF is defined as the Spectral Correlation Function (SCF). SCF can be measured by the normalized correlation between two spectral components of $x(t)$ at $f+\frac{\alpha}{2}$ and $f-\frac{\alpha}{2}$ over $\Delta t$ interval. Then, the ideal measurement of SCF can be expressed as:

$$
\begin{array}{r}
S_{X}^{\alpha}(f)=\lim _{T \rightarrow \infty} \lim _{\Delta t \rightarrow \infty} \frac{1}{\Delta t} \int_{-\Delta t / 2}^{\Delta t / 2} \frac{1}{T} X_{T}\left(t, f+\frac{\alpha}{2}\right) \\
X_{T}^{*}\left(t, f-\frac{\alpha}{2}\right) d t
\end{array}
$$

where finite time Fourier transform of $x(t)$ is

$$
X_{T}(t, f)=\int_{t-T / 2}^{t+T / 2} x(u) e^{j 2 \pi f u} d u
$$

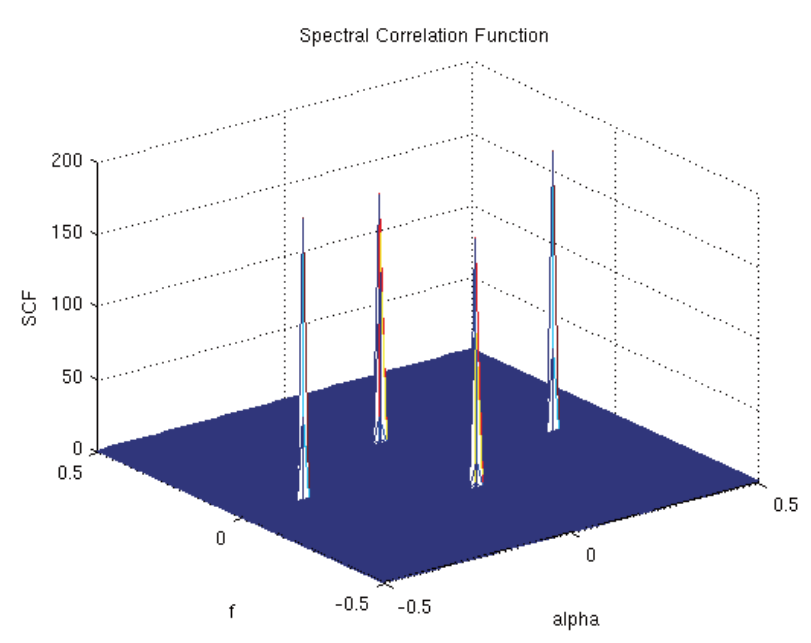

Fig. 1. The SCF of BPSK Modulation

Fig. 1 illustrates the theoretical SCF of a BPSK modulated signal. From this figure, we can observe that there are four peaks in the frequency-cyclic frequency domain. Two of the peaks appear in the frequency axis where $\alpha=0$ and $f=$ $\pm f_{c}$, and other two appear in the cyclic frequency axis where $f=0$ and $\alpha= \pm 2 f_{c}$. It is obvious that when $\alpha=0$ the SCF reduces to the power spectrum density (PSD) of the signal. It is important to note that Fig. 1 is generated from a large number of data. In reality, we can only obtain a limited length of signal to perform SCF.

To remove the channel effect, a normalized version of the SCF called the Spectral Coherence Function (SOF) can be generated as:

$$
C_{X}^{\alpha}(f)=\frac{S_{X}^{\alpha}(f)}{\left[S_{X}^{0}\left(f+\frac{\alpha}{2}\right)^{*} S_{X}^{0}\left(f-\frac{\alpha}{2}\right)\right]^{1 / 2}}
$$

It can be easily shown that the channel effects are removed from the $\mathrm{SOF}$, and the SOF is preserved as a reliable feature for parameter estimation and signal identification.

\section{B. System Model of Underwater Acoustic Communication Signal}

The transmitted signal of a single carrier underwater communication system corresponds to

$$
s(t)=\sum_{i=-\infty}^{\infty} b_{i} p(t-i T) \cos \left(2 \pi f_{c} t\right)
$$

where $b_{i}$ is the $i^{\text {th }}$ data symbol $\left(b_{i} \in\{+1,-1\}\right.$ for BPSK modulation), $p(t)$ is the pulse shape, $T$ is the symbol duration, and $f_{c}$ is the carrier frequency. At receiver side, the received signal experiences multi-path fading, Doppler shift, phase noise, and additive white Gaussian noise:

$$
\begin{array}{r}
r(t)=\Re\left\{\sum_{l=0}^{L-1} \sum_{i=-\infty}^{\infty} \alpha_{l}(t) e^{j \theta_{l}(t)} b_{i} p\left(t-i T-\tau_{l}(t)\right)\right. \\
\left.\cdot e^{j 2 \pi\left(f_{c}+\Delta f(t)\right)\left(t-\tau_{l}(t)\right)}\right\}+n(t)
\end{array}
$$

where $L$ is the maximum number of multi-path components, $l$ is the index for multi-path component, $\alpha_{l}$ is the fading gain of the $l^{\text {th }}$ path, $\theta_{l}$ is the phase offset introduced by the channel on the $l^{t h}$ path, $\tau_{l}$ is the time delay of the $l^{t h}$ path, $\Delta f$ is the Doppler shift, and $n(t)$ corresponds to the additive white Gaussian noise. Note that the fading, Doppler shift and phase noise are all functions of time.

If we calculate the SCF of the underwater acoustic communication signal over a long period of time, the varying multipath fading, Doppler shift and phase noise would distort the cyclic frequency features in the SCF, making the parameter estimation impossible to achieve. Hence, we have to apply a time domain window onto the received signal to obtain a short length of received signal so that within this length the channel and Doppler shift can be considered stable. Mathematically, this truncated signal corresponds to 


$$
\begin{aligned}
r_{0}(t)= & r(t) \cdot w(t) \\
= & \Re\left\{\sum_{l=0}^{L-1} \sum_{i=-\infty}^{\infty} \alpha_{l} e^{j \theta_{l}} b_{i} p\left(t-i T-\tau_{l}\right)\right. \\
& \left.\cdot e^{j 2 \pi\left(f_{c}+\Delta f\right)\left(t-\tau_{l}\right)}\right\}+n(t), \quad 0 \leq t \leq T_{w}
\end{aligned}
$$

where $T_{w}$ is the length of the rectangular window $w(t)$. In this article we assume the length $T_{w}$ is 0.125 second and it is consistent to the literature.

\section{Two-Stage Parameter Estimation}

The cyclic frequency features in SCF and SOF are discrete spectral lines, hence requiring very high resolution to observe them. To relieve the computational burden, we propose a twostage parameter estimation method and a dynamic varying resolution SCF/SOF algorithm. As shown in Fig. 2, a spectral analysis based coarse parameter estimation is first performed to obtain a coarse estimation of carrier frequency $\hat{f}_{c}$ and symbol rate $\hat{f}_{b}$ (where $f_{b}=1 / T$ ). Next, a cyclostationary analysis based estimation algorithm is used to obtain high resolution estimation $\hat{\hat{f}}_{c}$ and $\hat{\hat{f}}_{b}$.

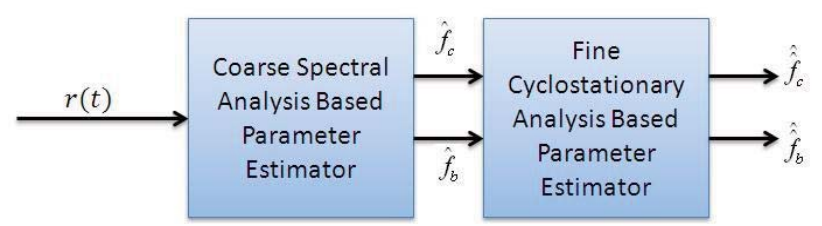

Fig. 2. Block Diagram of Parameter Estimation Algorithm

\section{A. Coarse Estimation via Spectral Analysis}

In the coarse estimation stage, we first obtain the spectrogram of the signal. Fig. 3 shows the spectrogram of a real underwater communication signal with carrier frequency $f_{c}=17000 \mathrm{~Hz}$ and symbol rate $f_{b}=4000 \mathrm{~Hz}$. Let $B(f, t)$ denote the spectrum at frequency $f$ and time $t$.

It is clear that the signal of interest is contained in the red rectangular area centered at $f_{c}$, with bandwidth approximately the vertical width of the red area. There exist some interferences and distortions out of the red rectangular area. To coarsely estimate the $f_{c}$ and the $f_{b}$, we

1) Find the maximal and minimal levels of the spectrum for different times $t$ :

$$
\begin{aligned}
P(t) & =\max _{f}|B(f, t)| \\
L(t) & =\min _{f}|B(f, t)|
\end{aligned}
$$

2) Find the threshold $T h_{1}$ to highlight out the red area in this figure. For example, we can set

$$
T h_{1}=\bar{L}+0.02 *(\bar{P}-\bar{L})
$$

where $\bar{L}$ and $\bar{P}$ denote the time average value of $L(t)$ and $P(t)$, respectively.
3) Update the spectrum by setting the spectrum values to be zero if the magnitude of the spectrum is less than the threshold. The interested area (the red rectangular area containing the signal in Fig. 3) can be expressed as the spectrum which has the frequency in the range $\left[\bar{f}_{\text {min }}, \bar{f}_{\text {max }}\right]$, shown in Fig. 4 .

4) Estimate the $\hat{f}_{c}$ and $\hat{f}_{b}$ :
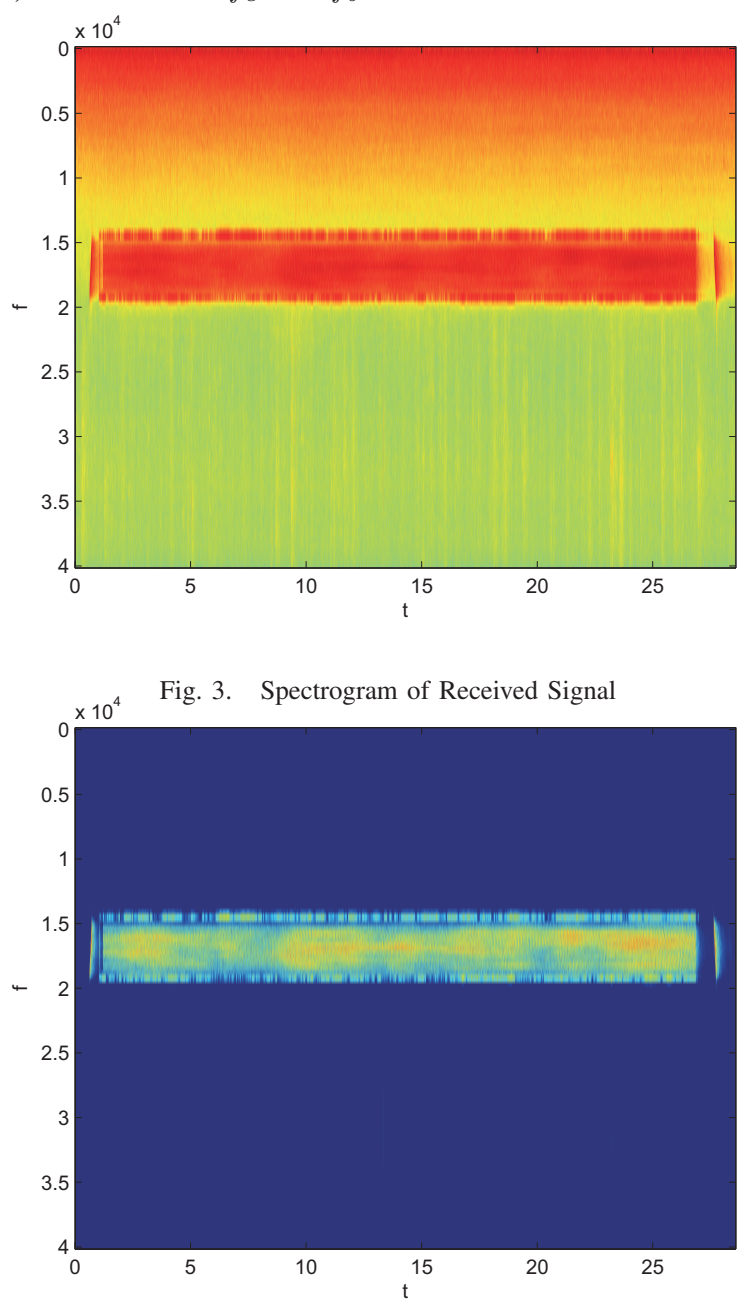

Fig. 4. Parameter Estimation via Spectrogram

- Find the highest frequency $f_{\max }$ for non-zero spectrum:

$$
f_{\text {max }}(t)=\max _{f}\{f: B(f, t) \mid>0\}
$$

- Computer the estimated highest frequency bound $\bar{f}_{\max }$ as the time average of $f_{\max }(t)$.

- Find the lowest frequency bound as:

$$
\bar{f}_{\text {min }}=\max _{f}\left\{f \mid f \in\left[0, \bar{f}_{\text {max }}\right], B(f, t) \leq 0.5 T h_{1}\right\}
$$

- Obtain coarse estimates $\hat{f}_{c}$ and $\hat{f}_{b}$ as:

$$
\begin{array}{r}
\hat{f}_{c}=\frac{1}{2}\left(\bar{f}_{\max }+\bar{f}_{\min }\right) \\
\hat{f}_{b}=\bar{f}_{\max }-\bar{f}_{\min }
\end{array}
$$




\section{B. Fine Estimation via Dynamic Varying Resolution Cyclosta- tionary Analysis}

With the coarsely estimated carrier frequency and symbol rate $\hat{f}_{c}$ and $\hat{f}_{b}$, we propose to use a dynamic varying resolution $\mathrm{SCF} / \mathrm{SOF}$ algorithm to estimate these parameters accurately. As seen in previous example, the cyclic frequency features we are interested in occur around $\alpha=0$ and $\alpha= \pm 2 f_{c}$. Hence, we don't need to calculate the whole SCF/SOF 3-d image with the same resolution. Instead, we only need to provide ultra-high resolution around cyclic frequency at $\alpha=0$ and $\alpha= \pm 2 f_{c}$ and use much more coarse resolution elsewhere. The algorithm is as follows:

1) Set the cyclic frequency resolution to be $d_{\alpha}=1 / T_{w}$ (which is the highest cyclic frequency resolution available given the window length of $\left.T_{w}\right)$ in $\left[2 \hat{f}_{c}-\Delta \alpha \leq\right.$ $\left.\alpha \leq 2 \hat{f}_{c}+\Delta \alpha\right]$ and $\left[\hat{f}_{b}-\Delta \alpha \leq \alpha \leq \hat{f}_{b}+\Delta \alpha\right]$, and $d_{\alpha}=0.01$ elsewhere.

2) Calculate the SCF and SOF with the varying resolution along the cyclic frequency axis.

3) Find the peaks in the cyclic frequency of SOF, the location of these peaks are high resolution estimation of carrier frequency and symbol rate, $\hat{\hat{f}}_{c}$ and $\hat{\hat{f}}_{b}$.

\section{NUMERICAL RESUlTS}

Now we use direct-sequence code-division-multiple-access (DS-CDMA) data [12] to validate the effectiveness of the proposed algorithm. The transmitted signal has a carrier frequency of $f_{c}=17,000 \mathrm{~Hz}$ and symbol rate of $f_{b}=4,000 \mathrm{~Hz}$ (example 1 ) or $f_{b}=4,000 \mathrm{~Hz}$ (example 2). The transmitted signal has a near flat spectrum but the received signal shows a spiky spectrum (Fig. 5 and Fig. 8) due to multipath interferences.

Example 1: Fig. 5 plots the power spectral density of received signal. In the PSD of the received signal, since there is no spectral line at carrier frequency, the peak occurs randomly at $16,830 \mathrm{~Hz}$, or the Doppler shift estimated being $-170 \mathrm{~Hz}$, which is a magnitude away from the correct value of $-15 \mathrm{~Hz}$.

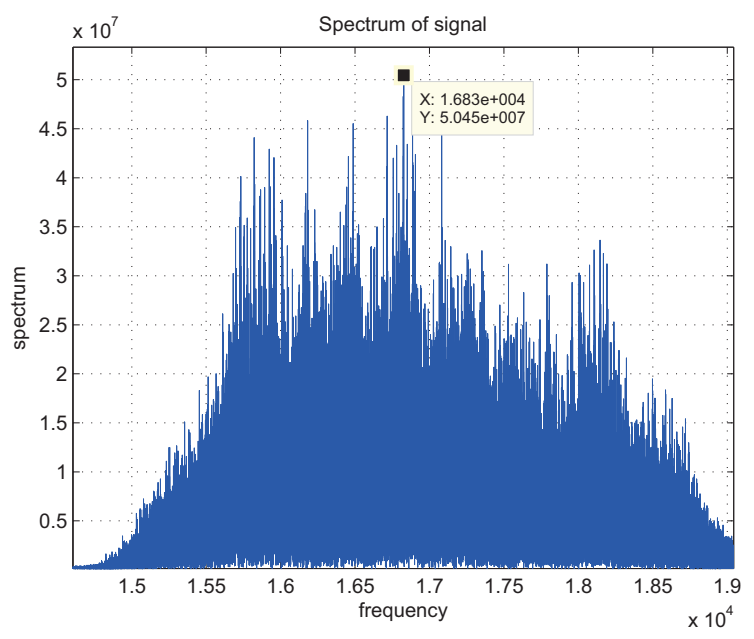

Fig. 5. Example 1: PSD of the received signal
Fig. 6 illustrates the high resolution SOF around $\alpha=2 f_{c}$. As shown in this figure, the SOF observes a spectral line at $33,970 \mathrm{~Hz}$. Hence, the carrier frequency can be estimated as $33970 / 2=16985 \mathrm{~Hz}$ and Doppler shift can be estimated as $-15 \mathrm{~Hz}$ which is the correct value.

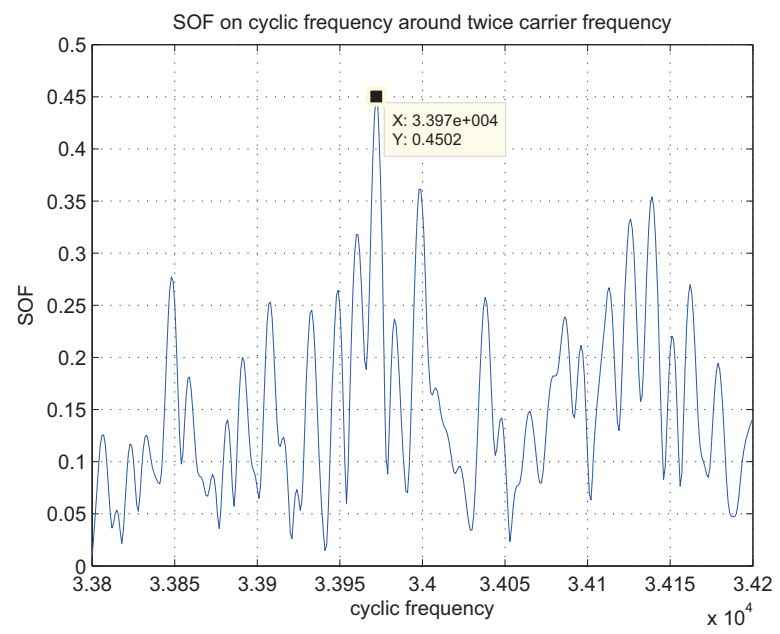

Fig. 6. Example 1: SOF of the received signal around twice the carrier frequency

Fig. 7 shows the high resolution $\mathrm{SOF}$ around $\alpha=f_{b}$. As expected, the SOF also observes a spectral line at $4,000 \mathrm{~Hz}$ which is the correct symbol rate of the transmitted signal.

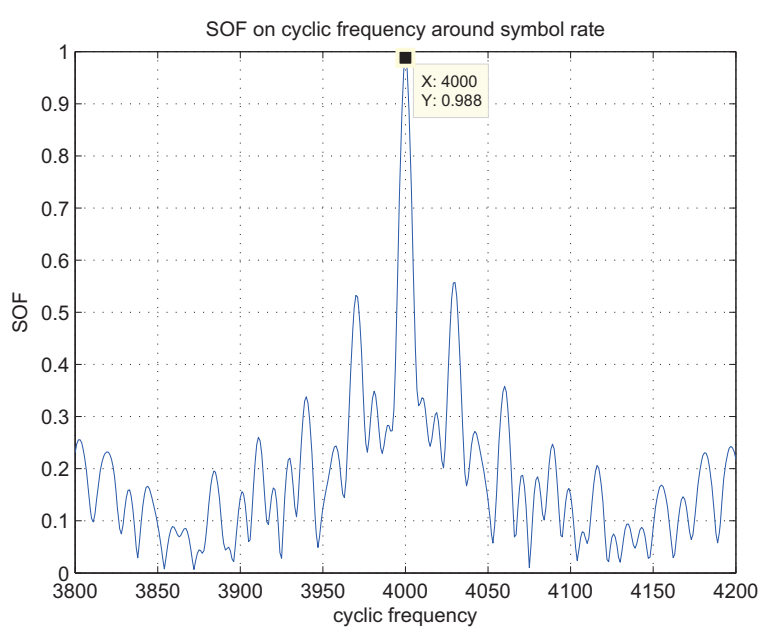

Fig. 7. Example 1: SOF of the received signal around the symbol rate

Example 2: Fig. 8 plots the power spectral density of received signal. In the PSD of the received signal, since there is no spectral line at carrier frequency, the peak occurs randomly at $17,330 \mathrm{~Hz}$. The Doppler shift is then estimated as $330 \mathrm{~Hz}$, which is a magnitude away from the correct value of $20 \mathrm{~Hz}$.

Fig. 9 illustrates the high resolution SOF around $\alpha=2 f_{c}$. As shown in this figure, the SOF observes a spectral line at $34,040 \mathrm{~Hz}$. Hence, the carrier frequency can be estimated as 


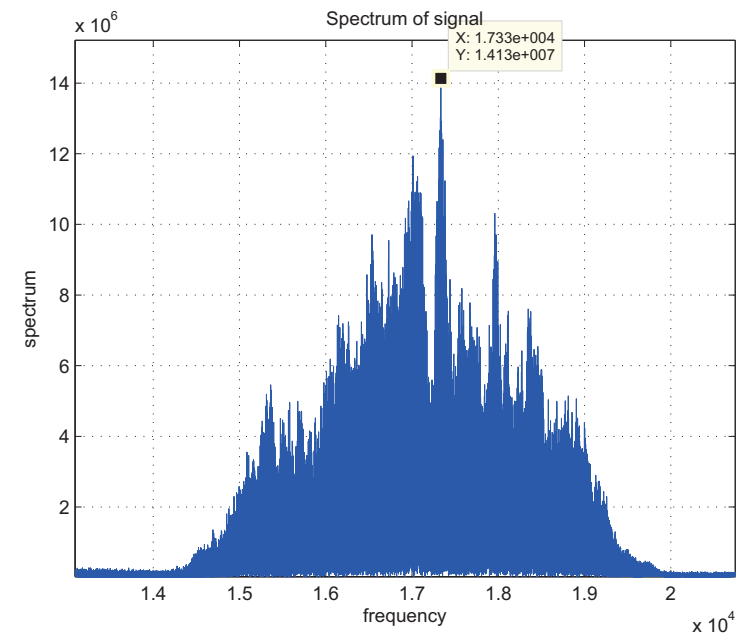

Fig. 8. Example 2: PSD of the received signal

$34040 / 2=17020 \mathrm{~Hz}$ and Doppler shift can be estimated as $20 \mathrm{~Hz}$ which is the correct value.

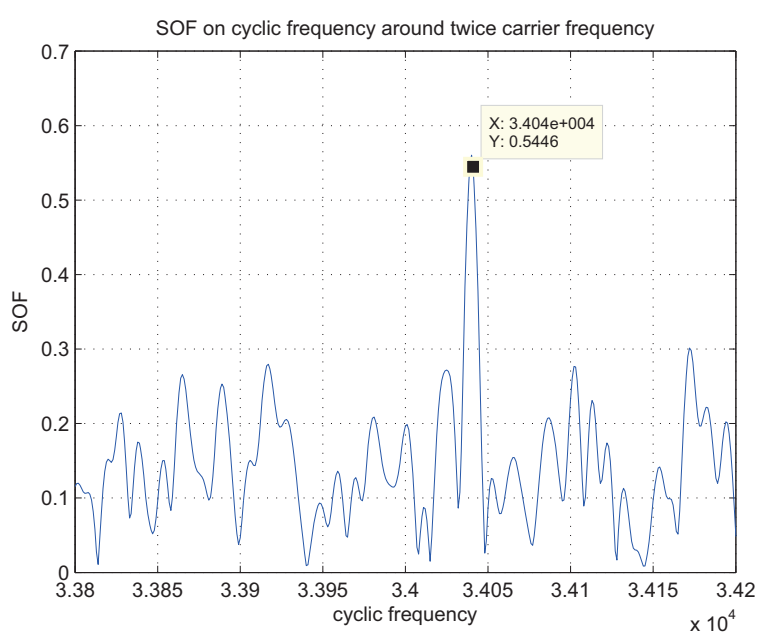

Fig. 9. Example 2: SOF of the received signal around twice the carrier frequency

Fig. 10 shows the high resolution SOF around $\alpha=f_{b}$. As expected, the SOF also observes a spectral line at $5,000 \mathrm{~Hz}$ which is the correct symbol rate of the transmitted signal.

\section{Conclusions}

Based on the second order cyclostationary features of the received signal, we proposed a new method to blindly estimate the carrier frequency (and corresponding Doppler shift) and symbol rate of underwater communication signals. The method does not require any a priori knowledge of the transmitted signal. Specifically, a two-stage estimation algorithm is developed: First, a coarse estimation based on spectral analysis is performed; Second, a fine estimation based on cyclostationary analysis is performed to obtain high resolution estimation

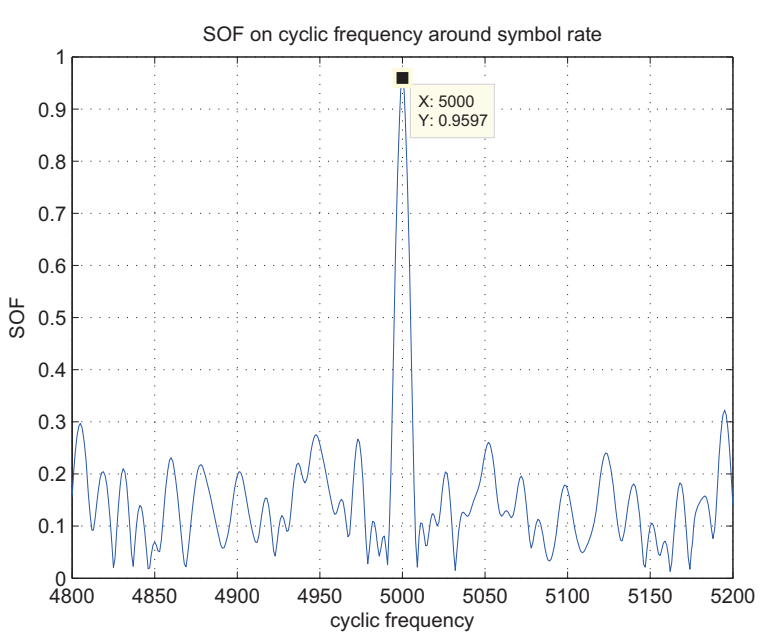

Fig. 10. Example 2: SOF of the received signal around the symbol rate

of carrier frequency and symbol rate. Taking advantage of the coarse estimation in the first stage, a dynamic varying resolution spectrum correlation function and spectrum coherence function generation is developed to significantly decrease the computational complexity by only calculating the SCF and SOF in cyclic frequency domain around the locations of expected features. Numerical results validate the effectiveness of the proposed method.

\section{REFERENCES}

[1] W. A. Gardner, Cyclostationarity in Communications and Signal Processing. IEEE Press,Piscataway, NJ, USA, 1993.

[2] W. A. Gardner, W. A. Brown, and C.-K. Chen, "Spectral correlation of modulated signalspart ii: digital modulation," IEEE Transactions on Communications, 1987.

[3] W. A. Gardner, "Signal interception: A unifying theoretical framework for feature detection," IEEE Transactions on Communications, 1988.

[4] A. Fehske, J. Gaeddert, and J. Reed, "A new approach to signal classification using spectral correlation and neural networks," in New Frontiers in Dynamic Spectrum Access Networks, 2005 (DySPAN 2005), pp. $144-150$, nov. 2005.

[5] M. Tsatsanis and G. Giannakis, "Blind estimation of direct sequence spread spectrum signals in multipath," IEEE Transactions on Signal Processing, vol. 45, pp. 1241-1252, may 1997.

[6] S. Buzzi and V. Massaro, "Parameter estimation and multiuser detection for bandlimited long-code cdma systems," IEEE Transactions on Wireless Communications, vol. 7, pp. 2307-2317, june 2008.

[7] W. Gardner, "Exploitation of spectral redundancy in cyclostationary signals," IEEE Signal Processing Magazine, vol. 8, pp. 14-36, apr 1991.

[8] E. Like, V. D. Chakravarthy, P. Ratazzi, and Z. Wu, "Signal classification in fading channels using cyclic spectral analysis," EURASIP Journal on Wireless Communications and Networking, vol. 2009, 2009.

[9] T. C. Yang, "Correlation-based decision feedback equalizer for underwater acoustic communications," in IEEE Journal of Oceanic Engineering, vol. 30, pp. 865-880, Oct. 2005.

[10] T. C. Yang and W.-B. Yang, "Performance analysis of direct-sequence spread spectrum underwater acoustic communications with low signalto-noise-ratio input signals," in J. Acoust. Soc Am., vol. 123(2), pp. 842855, Feb. 2008.

[11] G. Eynard and C. Laot, "Performance analysis of direct-sequence spread spectrum underwater acoustic communications with low signal-to-noiseratio input signals," in proc. of the IEEE OCEAN 2008, pp. 1-5, 2008.

[12] T. C. Yang, "Performance analysis of direct-sequence spread spectrum underwater acoustic communications with low signal-to-noise-ratio input signals," in J. Acoust. Soc Am., vol. 124(6), pp. 3632-3647, December 2008 . 